\title{
Questionnaire survey on adjuvant chemotherapy for elderly patients after gastrectomy indicates their vulnelabilities
}

\author{
Toshiyuki Tanahashi ${ }^{1} \cdot$ Kazuhiro Yoshida $^{1} \cdot$ Kazuya Yamaguchi $^{1} \cdot$ Naoki Okumura $^{2} \cdot$ Atsushi Takeno $^{3} \cdot$ \\ Kazumasa Fujitani ${ }^{4} \cdot$ Norimasa Fukushima $^{5} \cdot$ Nobuhiro Takiguchi $^{6} \cdot$ Yasunori Nishida $^{7} \cdot$ Narikazu Boku $^{8}$. \\ Takaki Yoshikawa $^{9} \cdot$ Masanori Terashima $^{10}$
}

Received: 11 January 2018 / Accepted: 3 May 2018 / Published online: 24 May 2018

(c) The International Gastric Cancer Association and The Japanese Gastric Cancer Association 2018

\begin{abstract}
Background In Japan, S-1 adjuvant chemotherapy for 1 year is the standard of care for the treatment of stage II and III patients under 80 years old with gastric cancer after curative operation. However, the feasibility of S-1 chemotherapy in patients over 80 years old has not yet been elucidated.

Methods To clarify the current treatment situation and feasibility of S-1 treatment in patients over 80 years old, a questionnaire survey of the patients treated from January 2011 to December 2012 was conducted at 58 member institutions of the Stomach Cancer Study Group of the JCOG (Japan Clinical Oncology Group).

Results Gastrectomy was performed in 15,573 patients of all ages, and 1,660 (10.7\%) patients were over 80 years of age. Of these elderly patients, $661(4.2 \%)$ were diagnosed as stage II and III. While S-1 adjuvant chemotherapy was recommended to $248(37.5 \%)$ of the stageII/III patients, only $99(15.0 \%)$ of them actually received S-1. Interestingly, the creatinine clearance rate was between 30 and $80 \mathrm{~mL} / \mathrm{min}$ in $87(87.9 \%)$ of the patients suggesting that S-1 dose modification should be considered. Moreover, S-1 compliance was poor in patients with more than $15 \%$ body weight loss.

Conclusion In general practice, surgery alone can be regarded as the standard of care for stage II and III gastric cancer patients over 80 years old. The feasibility and efficacy of S-1 adjuvant chemotherapy should be elucidated in a randomized control trial considering the vulnerabilities of the elderly.
\end{abstract}

Keywords Gastric cancer · Elderly patients · Adjuvant chemotherapy $\cdot$ S-1

\section{Introduction}

Gastric cancer (GC) is the third leading cause of cancer deaths in the world $(723,000$ deaths, $8.8 \%$ of the total) [1]. Almost one million new cases were estimated to have

Kazuhiro Yoshida

kyoshida@gifu-u.ac.jp

1 Department of Surgical Oncology, Gifu University Graduate School of Medicine, 1-1 Yanagido, Gifu 501-1194, Japan

2 Department of Surgery, Gifu Municipal Hospital, Gifu, Japan

3 Department of Surgery, Kansai Rosai Hospital, Amagasaki, Japan

4 Department of Surgery, Osaka Prefectural General Medical Center, Osaka, Japan

5 Department of Surgery, Yamagata Prefectural Central Hospital, Yamagata, Japan occurred in 2012, making it the fifth most common malignancy worldwide, with more than half of the cases occurring in Eastern Asian countries. Although it is the second leading cause of death from cancer, it remains the most frequently occurring cancer in Japan. In 1980, the peak of age-specific

6 Department of General Surgery, Chiba Cancer Center, Chiba, Japan

7 Department of Surgery, Keiyuukai Sapporo Hospital, Sapporo, Japan

8 Gastrointestinal Medical Oncology Division, National Cancer Center Hospital, Tokyo, Japan

9 Department of Gastrointestinal Surgery, Kanagawa Cancer Center, Yokohama, Japan

10 Division of Gastric Surgery, Shizuoka Cancer Center, Sunto, Japan 
incidence rate was around 75 years old, but the peak was even higher, at over 80 years old, in 2011 [2]. Therefore, the incidence rate of GC is increasing according to the age of the patients and its peak is shifting to the elderly.

According to the Annual Report for FY2015 from the Japan Cabinet Office, the number of people aged 65 and over is the highest ever, 31.90 million (30.79 million in 2012). The percentage of the population aged 65 years and over was $25.1 \%$ (24.1\% in 2012), and the population aged 75 years and over was 15.60 million, accounting for $12.3 \%$ of the total population [3]. Japan has become a super-aged society of the highest level and has the highest prevalence of GC in the world. Considering these facts, the number of elderly GC patients is expected to increase as this super-aged society progresses in the future [4].

In patients classified as having stage II or III GC according to the 13th edition of the Japanese Classification of Gastric Carcinoma [5], adjuvant chemotherapy consisting of S-1 monotherapy for 1 year after D2 gastrectomy is recommended by the Japanese guideline for the treatment of GC, based on the ACTS-GC trial [6], which was conducted in Japan for patients under the age of 80 years. However, it remains unclear whether S-1 should be administered to patients over 80 years of age after D2 gastrectomy for stage II and III GC. There is an urgent need to establish standard adjuvant treatment for elderly GC patients, especially those over 80 years old.

On the other hand, the elderly comprise a mixed population consisting of some healthy population similar to the younger population and others complicated with comorbidities such as cardiovascular, respiratory, mental, renal, and metabolic disease [7]. Interestingly, the European Organization for Research and Treatment of Cancer (EORTC) classified the elderly into three categories: (a) fit elderly patients who do not have any serious comorbidities and no dependence (Fit patients); (b) frail patients with significant dependency and comorbidities (Frail patients), and (c) patients with some instrumental activities of daily living dependency with or without severe comorbidity (Vulnerable patients) [8]. However, precise criteria or a definition of each category has not been clarified, and moreover, they might differ according to the target disease and treatment. Therefore, to conduct a randomized control trial (RCT) of adjuvant chemotherapy for elderly gastric cancer patients, certain definition classifying these three groups should be explored in the clinical practice. However, there have been no data on the safety and efficacy of S-1 in this population. Therefore, to clarify the current treatment situation, including compliance with S-1 adjuvant chemotherapy, and to identify the fit, frail and vulnerable population over 80 years old, a questionnaire survey was conducted among the member institutions of the Stomach Cancer Study Group of the Japan Clinical Oncology Group (JCOG).

\section{Methods and patients}

We conducted a questionnaire survey in 58 member institutions of the Stomach Cancer Study Group of the JCOG in October 2014 to evaluate the patients treated from January 2011 to December 2012. Packages including a cover letter and questionnaire documents were sent by e-mail to the chief of the department of each institution that described the research intent and provided a brief summary of the approved consensus for this survey by the Stomach Cancer Study Group at the JCOG meeting. Data collection was finalized by the end of April 2015.

The questionnaire consisted of the following seven questions: (Q1) How many gastrectomies were performed in your institution from January 2011 to December 2012? (Q2) How many patients were older than 80 years of age? (Q3) How many patients among them were diagnosed as pathological stage II and III according to the 14th edition of the Japanese Classification of Gastric Carcinoma [9]? (Q4) How many of the stage II/III patients over 80 years old did you recommend S-1 adjuvant chemotherapy? (Q5) If you did not recommend adjuvant chemotherapy to the patients over 80 years old, describe the reasons. The choices were 'too old', 'other cancer or disease', 'poor PS', 'poor oral intake', 'dimentia', 'no family support', and 'doctor's impression'. (Q6) If you recommended adjuvant chemotherapy to the patients over 80 years old, how many patients agreed and be actually prescribed S-1? (Q7) In reference to question 4 , how many patients did not agree to undergo adjuvant chemotherapy even if you recommended it, and describe the reasons why the patients did not agree. The choices were 'too old', 'adverse events', 'difficulty coming hospital', and 'economic reason'.

In addition to the above questions, we asked the doctors to provide information on the patients who were actually prescribed $\mathrm{S}-1$ as adjuvant chemotherapy, including age, sex, pathological stage, type of operation, method of reconstruction, date of surgery, surgery time, bleeding, extent of lymph node dissection, resection of other organs, the presence or absence of recurrence, co-morbidities as proposed by Charlson et al. [10], ECOG (Eastern Cooperative Oncology Group) performance status (PS), postoperative complications, body weight (BW) just before surgery and at the start of S-1, height, creatinine clearance rate (Ccr) as estimated by the Cockcroft-Gault equation at the start of S-1, date of the start of S-1, dead or alive, rate of continuation of S-1, dose and dosage schedule, and reasons for discontinuation. This study was approved by the investigational review board of the Gifu University Graduate School of Medicine and all other institutes. 


\section{Results}

\section{Number of patients treated by S-1 adjuvant chemotherapy over 80 years old}

We received replies from 51 member institutions (87.9\% response rate) of the Stomach Cancer Study Group of the JCOG. The total number of patients of all ages who underwent gastrectomy over the 2-year period was 15,573 , and among them, $10.7 \%$ (1660) were older than 80 years old (Fig. 1). Of the patients over 80 years old, 661 (4.2\%) were diagnosed as stage II (316) or III (345), and $37.5 \%$ (248, stage II: 98, stage III: 150) of the patients were recommended to receive S-1 adjuvant chemotherapy by a surgical and medical oncologist while 62.5\% (413, stage II: 218, stage III: 195) patients were not. One hundred five patients (stage II: 35, stage III:70) agreed and were actually prescribed S-1 as adjuvant chemotherapy after gastrectomy, and 143 patients (stage II: 63, stage III: 80 ) did not agree to undergo $\mathrm{S}-1$ adjuvant chemotherapy, despite the recommendation by their physician. In summary of the general practice, $15.0 \%$ (99) of the stage II and III patients older than 80 years old who were candidates for $S-1$ adjuvant chemotherapy in this survey actually received $S-1$ adjuvant chemotherapy, and the remaining patients received no treatment after gastrectomy, suggesting that surgery alone can be regarded as the standard of care in general practice for

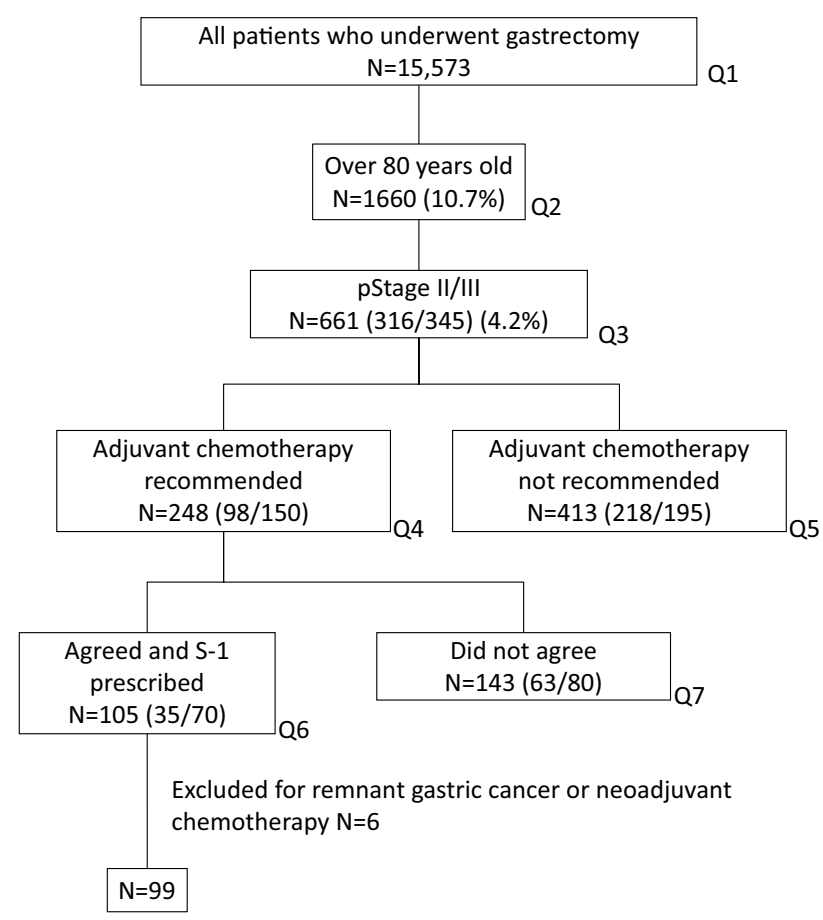

Fig. 1 Consort diagram of the number of patients evaluated and the related survey questions this category of patients in the member institutions of the Stomach Cancer Study Group of the JCOG.

\section{Reasons why S-1 was not recommended (Q5) and why the patients did not agree (Q7)}

It is quite important and interesting to investigate the reasons why S-1 was not administered in $556(413+143)$ of the patients because they involve two categories of patients: those for whom S-1 was not recommended (413) by doctors (Q5, doctor's reason) and those (143) for whom it was recommended, but who did not agree to take S-1 (Q7, patient's reason).

Concerning Q4, the most common reason for the physicians not to recommend a patient (413) undergo S-1 administration was that they regarded the patients as being very old (240) and, interestingly, the doctor's impression that the patients would not be able to continue the medication (69). Other reasons were that the patients had other disease (100) or other cancer (19), poor PS (65), poor oral intake (37), dementia (30), no family support (8), unknown (9), and miscellaneous reasons such as surgical complications (21).

The reasons why the patients (143) did not agree to undergo $\mathrm{S}-1$ administration even if it had been recommended (Q7) were that the patients regarded themselves as being very old (88), that they felt that they would not tolerate adverse events (42), and that they would not come to the hospital regularly (6). Interestingly, no patients refused to take $\mathrm{S}-1$ because of economic reasons. Other reasons included dementia, family's refusal, and poor PS.

\section{Compliance with S-1 in the elderly patients}

To clarify the patients older than 80 years of age for whom the administration of S-1 is feasible, clinicopathological data and compliance with S-1 therapy were compared. Especially, we focused on Ccr and BW loss after surgery. We excluded five patients who underwent surgery for remnant stomach cancer and one patient who underwent neoadjuvant chemotherapy in the present analysis; thus, 99 of the 105 patients were analyzed.

The characteristics of the 99 patients (63 men, 36 women) who were administered S-1 are shown in Table 1 . Their average age was 81.7 years old. Stage II cancer was present in 32 patients and stage III in 67 patients. Lymph node dissection was performed in each patient according to the $2010 \mathrm{Japa}-$ nese GC treatment guidelines [11].

Very interestingly, at the initial dosing of S-1, 66.7\% (66/99) of the patients were started at a lower than normal dose level (one level down: 54 patients, more than 2 levels down: 12 patients) by the doctor's decision. S-1 was administered at $40 \mathrm{mg} / \mathrm{m}^{2}$ of body surface area (BSA) twice daily every 6 weeks ( 4 weeks on and 2 weeks off). Patients with a 
Table 1 Characteristics of the 99 patients administered S-1

\begin{tabular}{|c|c|}
\hline & $N=99$ \\
\hline Age (mean) [range] & $81.7[80-90]$ years \\
\hline \multicolumn{2}{|l|}{ Sex } \\
\hline Male & 63 \\
\hline Female & 36 \\
\hline \multicolumn{2}{|l|}{ Pathological stage } \\
\hline Stage II & 32 \\
\hline Stage III & 67 \\
\hline \multicolumn{2}{|l|}{ Charlson Comorbidity Index } \\
\hline 0 & 59 \\
\hline 1 & 28 \\
\hline 2 & 10 \\
\hline 3 & 1 \\
\hline 6 & 1 \\
\hline \multicolumn{2}{|l|}{ Performance status } \\
\hline 0 & 60 \\
\hline 1 & 34 \\
\hline 2 & 5 \\
\hline \multicolumn{2}{|l|}{ Operation } \\
\hline Distal gastrectomy & 64 \\
\hline Total gastrectomy & 27 \\
\hline Others & 3 \\
\hline \multicolumn{2}{|l|}{ Reconstruction } \\
\hline Billroth I & 31 \\
\hline Billroth II & 5 \\
\hline Roux-en-Y & 55 \\
\hline Others & 8 \\
\hline \multicolumn{2}{|l|}{ Surgery } \\
\hline Time (mean/median) & $233.6 \mathrm{~min} / 216 \mathrm{~min}$ \\
\hline Bleeding (mean/median) & $300.9 \mathrm{~g} / 150 \mathrm{~g}$ \\
\hline \multicolumn{2}{|l|}{ Lymph node dissection } \\
\hline D1 & 5 \\
\hline D1+ & 11 \\
\hline D2 or over & 79 \\
\hline Unknown & 4 \\
\hline \multicolumn{2}{|l|}{ Resection of other organs } \\
\hline Yes & 28 \\
\hline No & 66 \\
\hline Unknown & 5 \\
\hline \multicolumn{2}{|l|}{ Recurrence } \\
\hline Yes & 33 \\
\hline No & 65 \\
\hline Unknown & 1 \\
\hline \multicolumn{2}{|l|}{ Prognosis } \\
\hline Alive & 66 \\
\hline Dead & 28 \\
\hline Unknown & 5 \\
\hline
\end{tabular}

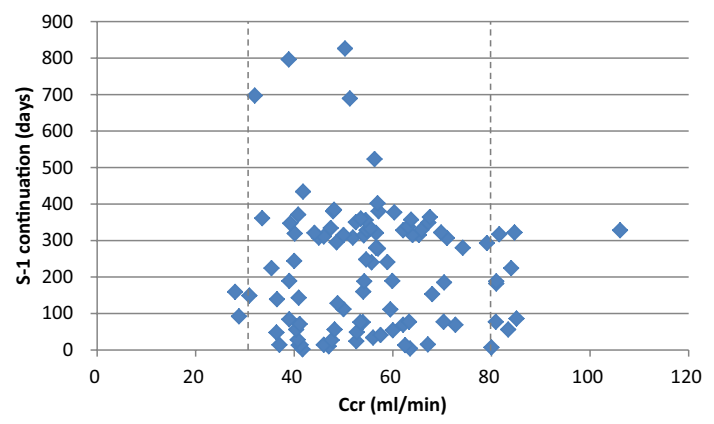

\begin{tabular}{|c|c|c|c|c|}
\hline \multirow{2}{*}{ Ccr $(\mathrm{mL} / \mathrm{min})$} & $<30$ & \multicolumn{2}{|c|}{$\geq 30$ to $<80$} & \multirow{2}{*}{$\geq 80$} \\
\cline { 3 - 4 } & & $\geq 30$ to $<60$ & $\geq 60$ to $<80$ & \multirow{2}{*}{10} \\
\hline \multirow{2}{*}{ Number } & 2 & \multicolumn{2}{|c|}{$87(87.9 \%)$} & \\
\cline { 3 - 4 } & & 64 & 23 & \\
\hline
\end{tabular}

Fig. 2 Correlation between continuation of S-1 chemotherapy and creatine clearance rate $(\mathrm{Ccr})$

BSA of $<1.25 \mathrm{~m}^{2}$ received $80 \mathrm{mg}$ daily; those with a BSA of $\geq 1.25 \mathrm{~m}^{2}$ but $<1.5 \mathrm{~m}^{2}$ received $100 \mathrm{mg}$ daily; and those with a BSA of $\geq 1.5 \mathrm{~m}^{2}$ received $120 \mathrm{mg}$ daily [6]. The daily decrease in dosage level was carried out as follows: from 120 to $100 \mathrm{mg}, 100-80 \mathrm{mg}$, or $80-50 \mathrm{mg}$. No modifications in the treatment schedule were conducted in 33 of the 99 patients analyzed at the initial administration: $61.6 \%(61 / 99)$ of the patients started treatment for 4 weeks on and 2 weeks off, whereas 33 patients were treated 2 weeks on and 1 week off.

To assess the 1-year compliance with S-1 chemotherapy, 77 of the 99 patients were analyzed. The remaining 22 patients, in whom cancer recurred or who died within 1 year after surgery, were excluded. Administration of S- 1 for 1 year was completed in $44.2 \%$ (34/77) of the patients, and $59.7 \%$ (46/77) of the patients were successfully treated for more than 6 months with variations in dose modification.

In the next analysis, Ccr (as estimated by the CockcroftGault equation) and compliance with S-1 administration were assessed in the 99 patients including recurrent cases as shown in Fig. 2. According to the S-1 administration guidelines, standard administration is recommended in patients with a Ccr of $>80 \mathrm{~mL} / \mathrm{min}$, dose modification should be considered in patients with a Ccr between 30 and $80 \mathrm{~mL} /$ min, and $\mathrm{S}-1$ should not be administered in patients with a $\mathrm{Ccr}$ of $<30 \mathrm{~mL} / \mathrm{min}$. The patients were divided into these three categories and compliance with S-1 was compared. All two patients who had a $\mathrm{Ccr}$ of $<30 \mathrm{~mL} / \mathrm{min}$ could not continue S-1 for more than 6 months. Of the 10 patients with a Ccr of $>80 \mathrm{~mL} / \mathrm{min}$, three patients completed the 1 -year therapy, five patients continued it for 6 months, and cancer recurred in two patients within 1 year. Very interestingly, the Ccr of $87.9 \%$ (87/99) of the patients was between 30 and $80 \mathrm{~mL} / \mathrm{min}$, which strongly suggests that dose modification 
should be considered for elderly patients undergoing S-1 administration. In this population, administration of $\mathrm{S}-1$ for 1 year was completed in $42.5 \%$ (38/87) of the patients, and $59.8 \%(52 / 87)$ of the patients were successfully treated for more than 6 months with variations in dose modification. $72.4 \%(63 / 87)$ began with dose modification from the initial treatment. There was no significant correlation between Ccr and compliance with S-1 administration in the present analysis.

Another important factor relating to compliance with S-1 is BW loss after surgery as reported by Aoyama et al. [12]. According to their report, BW loss of $>15 \%$ at 1 month after surgery, not the pre-operative BW loss, was a significant risk factor by both univariate and multivariate analyses. In the present analysis, we divided the patients into three categories, with BW loss defined as (preoperative BW-BW at the start of S-1) $\times 100 /$ preoperative BW: $>15 \%$ (9 patients), $10-15 \%$ (26 patients), and $<10 \%$ (64 patients) compared to the preoperative BW (Table 2). The 1-year continuation rate was $43.8 \%$ in the patients with $<10 \% \mathrm{BW}$ loss, $46.2 \%$ in those with $10-15 \%$ BW loss, and $11.1 \%$ in those with $>15 \%$ BW loss. The 6-month continuation rates were 57.8, 69.2, and $33.3 \%$, respectively, suggesting that BW loss of $>15 \%$ may be one of the risk factors for poor S-1 compliance.

\section{Discussion}

Tremendous discussions have taken place to determine whether adjuvant chemotherapy should be administered to stage II and III GC patients over 80 years old. The ACTSGC trial demonstrated that the rate of overall survival and recurrence-free survival of stage II and III GC patients under 80 years of age were much improved when these patients were treated by S-1 for 1 year after curative operation compared to surgery alone [13]. Currently in Japan, a clinical trial to determine the optimal period of adjuvant S-1 chemotherapy for pathological stage II GC patients who underwent D2 gastrectomy(JCOG1104) has just finished. It was concluded in ESMO 2017 that postoperative S-1 adjuvant chemotherapy for p-stage II GC should be continued until 1 year so far as feasible. Another trial, a phase III study of docetaxel plus S-1 [14] versus S-1 alone in the treatment of curatively resected stage III GC, is ongoing. To establish a

Table 2 Correlation between S-1 continuation rate and body weight loss

\begin{tabular}{llll}
\hline Body weight loss & $<10 \%$ & $\geq 10 \%$ to $<15 \%$ & $\geq 15 \%$ \\
Number & 64 & 26 & 9 \\
1-year continuation rate & $43.8 \%$ & $46.2 \%$ & $11.1 \%$ \\
6-month continuation rate & $57.8 \%$ & $69.2 \%$ & $33.3 \%$ \\
\hline
\end{tabular}

general consensus on this issue involving elderly patients, a RCT should be conducted in the era of evidence-based medicine. However, no solid data or baseline data for the assessment of safety and efficacy in patients over 80 years old is available for constructing a protocol for the RCT. The present study was conducted to acquire basic information concerning patients over 80 years old, especially information on the feasibility of S-1 adjuvant chemotherapy and a general consensus on this topic in daily practice in highvolume member institutions of the Stomach Cancer Study Group of the JCOG.

Respondents to the present questionnaire survey voiced controversial opinions, either that elderly patients should be treated with S-1 because of the solid data from the ACTSGC trial, or that they should not be treated because with their physical and/or mental comorbidities, elderly patients are too old to tolerate chemotherapy with/without the support of their families. This was probably because the respondents had not yet considered that the elderly comprise a heterogeneous population of Fit patients, Frail patients, and Vulnerable patients, as classified by EORTC [8]. Based on this classification, even in the elderly, the Fit patients are able to receive the same therapy, so that $\mathrm{S}-1$ adjuvant chemotherapy can be administered to Fit patients after gastrectomy in the same manner as that in the ACTS-GC trial. This strategy can be ethically acceptable. At the same time, such treatment should be avoided in Frail patients. They would be poor PS, poor oral intake, dementia and no family support. However, a concept should be established for Vulnerable patients when establishing a standard treatment for the elderly. At present, there is no scientific consensus on this category, and clear eligibility criteria for these Vulnerable patients urgently requires elucidation.

The Comprehensive Geriatric Assessment (CGA) is an approach developed and used by geriatricians to set up individualized and proactive patient care plans. The CGA estimates a patient's functional status, the presence of comorbidities [10], mental status [15] and emotional condition [16], social support, nutritional status [17], polypharmacy [18], and the presence or absence of geriatric syndromes [19, 20]. Especially, in a trial of adjuvant chemotherapy, patients were eligible for curative operations and were ready for rehabilitation and to resume social activities. In this sense, a new and unique strategy along with the CGA for considering S-1 administration as adjuvant chemotherapy in Vulnerable patients is warranted.

We have focused on three aspects in considering the vulnerability of stage II and III GC patients over 80 years of age to undergo S-1 administration as adjuvant chemotherapy. First, we regard the Ccr as the most important factor for determining S-1 administration, especially for setting the $\mathrm{S}-1$ dose. A Ccr of $<60 \mathrm{~mL} / \mathrm{min}$ has been reported to be a significant risk factor for 6-month continuation of S-1 
adjuvant chemotherapy [21]. However, in our survey, there was no significant correlation between the value of $\mathrm{Ccr}$ and the 1-year oral S-1 continuation rate. The 1-year and 6-month continuation rates were $44.2 \%$ (33/77) and 59.7\% (46/77), respectively, and these rates were quite reasonable if we consider that the 1-year continuation rate of patients aged 75-80 years in the ACTS-GC trial was $48.9 \%$ (unpublished data). This might have occurred because in 66 of the 99 patients, the initial dose of S-1 was reduced from the standard regimen considering the $\mathrm{Ccr}$ of the patients. The $\mathrm{Ccr}$ in $87.9 \%$ of the patients was between 30 and $80 \mathrm{~mL} / \mathrm{min}$. Moreover, according to the S-1 post-marketing use results survey, the incidence of Grade 3 and 4 adverse events in patients with a Ccr between 30 and $80 \mathrm{~mL} / \mathrm{min}$ was $7.8 \%$ higher than that of patients with a Ccr of $\geq 80 \mathrm{~mL} / \mathrm{min}$. The major reason of S-1 discontinuation was adverse events, such as appetite loss, general fatigue and neutropenia. Therefore, $\mathrm{Ccr}$ can be considered one of the important components marking the vulnerability of the elderly to $\mathrm{S}-1$ administration. Of course, for patients with a Ccr of $<30 \mathrm{~mL} / \mathrm{min}$ (Frail patients), S-1 adjuvant chemotherapy would not be the best choice.

Second, BW loss has been regarded as the most important risk factor for compliance with S-1 adjuvant chemotherapy, as shown by Aoyama et al. [12]. In our survey, a BW loss of $>15 \%$ also led to a very poor continuation rate and as a result, this population can be regarded as Frail patients.

Third, we believe that the type of operation performed might affect compliance with the oral administration of chemotherapy. Tsujimoto et al. showed that the completion rate of S-1 treatment did not depend on the type of surgical procedure, i.e., gastrectomy, reconstruction, or resection of other organs [22]. However, patients undergoing total gastrectomy generally experience greater BW loss compared to those undergoing distal gastrectomy [23]. This BW loss in the elderly can affect the compliance with oral S-1 after surgery. In fact, in the present survey, the S-1 continuation rate of the patients with total gastrectomy tended to be lower than that of the patients with non-total gastrectomy. Table 3 defines the candidate Vulnerable patients for S-1 administration as adjuvant chemotherapy considering the Ccr, BW loss, and type of operation as listed above. Patients with a Ccr of $<30 \mathrm{~mL} / \mathrm{min}$ and BW loss of $>15 \%$ can be regarded as Frail patients, whereas those with a Ccr of $>80 \mathrm{~mL} / \mathrm{min}$ undergoing a non-total gastrectomy should be considered Fit, and patients undergoing other categories of non-total and total gastrectomy can be considered Vulnerable patients in the ongoing clinical trials. Moreover, the CGA are important factors for evaluating the vulnerability of the elderly patients. These tools should be assessed for patients in the RCT.

There are some limitations in the present survey. First, this is a questionnaire-based survey of a retrospective cohort method. The data from the surveyed institutions might
Table 3 The categories of fit, frail and vulnerable patients

\begin{tabular}{|c|c|c|c|}
\hline & \multicolumn{3}{|c|}{$\operatorname{Ccr}(\mathrm{mL} / \mathrm{min})$} \\
\hline & $<30$ & $\geq 30$ to $<80$ & $\geq 80$ \\
\hline \multicolumn{4}{|c|}{ Non-total gastrectomy } \\
\hline BW loss $<15 \%$ & Frail & Vulnerable & Fit \\
\hline BW loss $\geq 15 \%$ & Frail & Frail & Frail \\
\hline \multicolumn{4}{|l|}{ Total gastrectomy } \\
\hline BW loss $<15 \%$ & Frail & Vulnerable & Vulnerable \\
\hline $\mathrm{BW}$ loss $\geq 15 \%$ & Frail & Frail & Frail \\
\hline
\end{tabular}

$B W$; body weight, $C c r$; creatinine clearance

not necessarily represent the vast majority of community practice and might include selection bias. However, to our knowledge, this is the first report of a survey of postoperative adjuvant chemotherapy in patients older than 80 years, and the findings might help in constructing concepts for a future trial to investigate this issue. Second, Moertal et al. showed that patient choice influences outcome with his study showing patients declining randomisation actually doing better than those receiving chemotherapy [24]. Since the present data are from other facilities and retrospective survey, not randomization, we could not investigate patients' belief for chemotherapy at that time.

In estimating the candidate patients who could be enrolled into a future study, among the 661 candidate patients for S-1 adjuvant chemotherapy in this survey, S- 1 therapy was recommended in $37.5 \%$ (248) of the patients, but not recommended in the remainder (413) by doctor's decision based on the patient's comorbidities and/or vague reasons that the patients were very old. Moreover, among the 248 patients for whom S-1 was recommended, 143 patients rejected the treatment. Therefore, when considering a RCT for this issue, a reasonable number of the patients can be considered candidates for participation among the present population $(413+143)$. The effect of S-1 may be diminished by starting with dose reduction. A phase III trial to confirm S-1 adjuvant chemotherapy for pathological stage II/III GC in vulnerable elderly patients who have undergone gastrectomy is just started in the JCOG.

\section{Conclusion}

In general practice, surgery alone can presently be regarded as the standard of care for stage II and III GC patients over 80 years old. The feasibility and efficacy of S-1 adjuvant chemotherapy should be further elucidated by RCTs considering the vulnerabilities of the elderly.

Acknowledgements The authors wish to acknowledge to all members and facilities who answered the questionnaire survey in the Stomach 
Cancer Study Group of the JCOG.: Aichi Cancer Center Hospital, Cancer Institute Hospital, Chiba Cancer Center, Fukuyama City Hospital, Gifu Municipal Hospital, Gifu University School of Medicine, Hakodate Goryoukaku Hospital, Hiroshima City Asa Citizens Hospital, Hiroshima City Hiroshima Citizens Hospital, Hiroshima University Hospital, Hyogo Cancer Center, Hyogo College of Medicine, Ishikawa Prefectural Central Hospital, Itami City Hospital, Iwate Medical University, Kanagawa Cancer Center, Kansai Medical University Hospital, Kansai Rosai Hospital, Keiyukai Sapporo Hospital, Kindai University Faculty of Medicine, Kitasato University School of Medicine, Kobe University School of Medicine, Miyagi Cancer Center, Nagaoka Chuo General Hospital, Nagoya University Graduate School of Medicine, National Cancer Center Hospital, National Cancer Center Hospital East, National Hospital Organization Kyoto Medical Center, Niigata Cancer Center Hospital, Oita University Hospital, Okayama University Hospital, Osaka Prefectural General Medical Center, Osaka Medical Center for Cancer and Cardiovascular Diseases, Osaka Medical College, Osaka University Faculty of Medicine, Saitama Cancer Center, Saitama Medical University International Medical Center, Sakai City Medical Center, Sendai Medical Center, Shikoku Cancer Center, Shimane University Faculty of Medicine, Shizuoka Cancer Center, Shizuoka General Hospital, Tenri Hospital, Tochigi Cancer Center, Tokyo Medical and Dental University, Tokyo Metropolitan Cancer and Infectious Diseases Center Komagome Hospital, Toranomon Hospital, Toyama Prefectural Central Hospital, Wakayama Medical University, Yamagata Prefectural Central Hospital.

\section{Compliance with ethical standards}

Conflict of interest $\mathrm{K}$. Yoshida has received grant, personal fees and nonfinancial support from Taiho Pharm. Co., Ltd; Ono Pharm. Co., Ltd.; Chugai Pharmaceutical Co., Ltd.; Yakult Honsha Co., Ltd.; Eli Lilly Japan K.K.; Daiichi Sankyo Co., Ltd.; Merk Serono Co., Ltd.; Novartis Pharma; EA Pharma Co., Ltd.; Takeda Pharmaceutical Company; Sanofi K.K.; grant and personal fees from Johnson \& Johnson K.K.; Covidien Japan; grant from Nippon Kayaku Co., Ltd.; Otsuka Pharma Co., Ltd.; Dainippon Sumitomo Pharma; personal fees from MSD K.K.; Bayer Yakuhin, Ltd.N. Takiguchi has received grant from Ministry of Health, Labor and Welfare, Japan.N. Boku has received grant and personal fees from Taiho Pharm. Co., Ltd.T. Yoshikawa has received grant from Japanese Foundation for Multidisciplinary Treatment of Cancer; grant and personal fees from Chugai Pharmaceutical Co., Ltd.; Taiho Pharm. Co., Ltd; Noartis Pharma; personal fees from Abbott Japan; Ono Pharm. Co., Ltd.; Eli Lilly Japan K.K.; Kaken Pharmaceutical; Yakult Honsha Co., Ltd.; Nippon Kayaku Co., Ltd.; Johnson \& Johnson K.K.; Covidien Japan; Takeda Pharmaceutical Company; Olympus. All remaining authors declared that they have no conflict of interest.

Human rights statement and informed consent All the questions asked in this survey were about the preference of the clinical practice of each investigator. Any patient information such as names, initials, addresses, admission dates, hospital numbers, or any other data that might identify patients were not asked or included in this survey.

\section{References}

1. Ferlay J, Soerjomataram I, Dikshit R, Eser S, Mathers C, Rebelo $\mathrm{M}$, et al. Cancer incidence and mortality worldwide: sources, methods and major patterns in GLOBOCAN 2012. Int J Cancer. 2015;136:E359-86.
2. Cancer Information Service NCC, Japan., Cancer Registry and Statistics. 2016.

3. The Aging Society: Current Situation and Implementation Measures FY 2013.

4. Arai H, Ouchi Y, Toba K, Endo T, Shimokado K, Tsubota K, et al. Japan as the front-runner of super-aged societies: Perspectives from medicine and medical care in Japan. Geriatr Gerontol Int. 2015;15:673-87.

5. Japanese Gastric Cancer A. Japanese classification of gastric carcinoma-2nd English Edition. Gastric Cancer. 1998;1:10-24.

6. Sakuramoto S, Sasako M, Yamaguchi T, Kinoshita T, Fujii M, Nashimoto A, et al. Adjuvant chemotherapy for gastric cancer with S-1, an oral fluoropyrimidine. N Engl J Med. 2007;357:1810-20.

7. Stober J, Hong HG, Czado C, Ghosh P. Comorbidity of chronic diseases in the elderly: patterns identified by a copula design for mixed responses. Comput Stat Data Anal. 2015;88:28-39.

8. Pallis AG, Fortpied C, Wedding U, Van Nes MC, Penninckx B, Ring A, et al. EORTC elderly task force position paper: approach to the older cancer patient. Eur J Cancer. 2010;46:1502-13.

9. Assoc JGC. Japanese classification of gastric carcinoma: 3rd English edition. Gastric Cancer. 2011;14:101 - 12.

10. Charlson ME, Pompei P, Ales KL, MacKenzie CR. A new method of classifying prognostic comorbidity in longitudinal studies: development and validation. J Chronic Dis. 1987;40:373-83.

11. Japanese Gastric Cancer A. Japanese gastric cancer treatment guidelines 2010 (ver. 3). Gastric Cancer. 2011;14:113-23.

12. Aoyama T, Yoshikawa T, Shirai J, Hayashi T, Yamada T, Tsuchida $\mathrm{K}$, et al. Body weight loss after surgery is an independent risk factor for continuation of S-1 adjuvant chemotherapy for gastric cancer. Ann Surg Oncol. 2013;20:2000-6.

13. Sasako M, Sakuramoto S, Katai H, Kinoshita T, Furukawa H, Yamaguchi T, et al. Five-year outcomes of a randomized phase III trial comparing adjuvant chemotherapy with $\mathrm{S}-1$ versus surgery alone in stage II or III gastric cancer. J Clin Oncol. 2011;29:4387-93.

14. Yoshida K, Hirabayashi N, Takiyama W, Ninomiya M, Takakura $\mathrm{N}$, Sakamoto J, et al. Phase I study of combination therapy with S-1 and docetaxel (TXT) for advanced or recurrent gastric cancer. Anticancer Res. 2004;24:1843-51.

15. Yesavage JA, Brink TL, Rose TL, Lum O, Huang V, Adey $\mathrm{M}$, et al. Development and validation of a geriatric depression screening scale: a preliminary report. J Psychiatr Res. 1982;17:37-49.

16. Folstein MF, Folstein SE, McHugh PR. "Mini-mental state". A practical method for grading the cognitive state of patients for the clinician. J Psychiatr Res. 1975;12:189-98.

17. Guigoz Y, Vellas B, Garry PJ. Assessing the nutritional status of the elderly: the mini nutritional assessment as part of the geriatric evaluation. Nutr Rev. 1996;54:S59-65.

18. Bellera CA, Rainfray M, Mathoulin-Pélissier S, Mertens C, Delva F, Fonck M, et al. Screening older cancer patients: first evaluation of the G-8 geriatric screening tool. Ann Oncol. 2012;23:2166-72.

19. Extermann M, Hurria A. Comprehensive geriatric assessment for older patients with cancer. J Clin Oncol. 2007;25:1824-31.

20. Carreca I, Balducci L, Extermann M. Cancer in the older person. Cancer Treat Rev. 2005;31:380-402.

21. Aoyama T, Yoshikawa T, Hayashi T, Kuwabara H, Mikayama $\mathrm{Y}$, Ogata T, et al. Risk factors for 6-month continuation of S-1 adjuvant chemotherapy for gastric cancer. Gastric Cancer. 2013;16:133-9. 
22. Tsujimoto H, Horiguchi H, Hiraki S, Yaguchi Y, Takahata R, Kumano I, et al. Tolerability of adjuvant chemotherapy with S-1 after curative resection in patients with stage II/III gastric cancer. Oncol Lett. 2012;4:1135-9.

23. Takachi K, Doki Y, Ishikawa O, Miyashiro I, Sasaki Y, Ohigashi $\mathrm{H}$, et al. Postoperative ghrelin levels and delayed recovery from body weight loss after distal or total gastrectomy. J Surg Res. 2006;130:1-7.

24. Moertel CG, Childs DS, O'Fallon JR, Holbrook MA, Schutt AJ, Reitemeier RJ. Combined 5-fluorouracil and radiation therapy as a surgical adjuvant for poor prognosis gastric carcinoma. J Clin Oncol. 1984;2:1249-54. 